\title{
Clinical characteristics and infectivity of asymptomatic carriers of SARS-CoV-2 (Review)
}

\author{
WANYING ZHANG, XUEJE WU, HUI ZHOU and FENG XU \\ Department of Infectious Diseases, The Second Affiliated Hospital, \\ Zhejiang University School of Medicine, Hangzhou, Zhejiang 310009, P.R. China
}

Received July 28, 2020; Accepted November 2, 2020

DOI: $10.3892 /$ etm.2020.9547

\begin{abstract}
Since December 2019, the spread of severe acute respiratory syndrome coronavirus 2 (SARS-CoV-2) has become a global pandemic. At present, confirmed patients are the main source of infection, while a number of studies have indicated that asymptomatic carriers also have the ability to spread the virus. As of September 29, 2020, as the first country to report coronavirus disease 2019 (COVID-19), China has 375 asymptomatic infections according to the National Health Commission of China. Asymptomatic carriers have become the current focus of global epidemic prevention and control efforts. The present review article provides a brief introduction on the clinical characteristics and infectivity of asymptomatic carriers, and makes suggestions for the identification of asymptomatic carriers.
\end{abstract}

\section{Contents}

1. Introduction

2. Demographic characteristics

3. Ratio of asymptomatic carriers among all infected persons

4. Radiological and laboratory data

5. Infectivity and transmission risk of asymptomatic carriers

6. Transmission period of asymptomatic carriers

7. Advice to identify asymptomatic carriers

8. Conclusions

\section{Introduction}

In December 2019, the first case of pneumonia of unknown etiology was identified in Wuhan, China (1). Subsequently,

Correspondence to: Dr Feng Xu, Department of Infectious Diseases, The Second Affiliated Hospital, Zhejiang University School of Medicine, 88 Jiefang Road, Hangzhou, Zhejiang 310009, P.R. China E-mail: xufeng99@zju.edu.cn

Key words: coronavirus disease 2019, asymptomatic carriers, clinical characteristics, infectivity the pathogen was isolated and officially named Severe Acute Respiratory Syndrome Coronavirus 2 (SARS-CoV-2) by the International Committee on Taxonomy of Viruses, due to its marked similarity with the agent of Severe Acute Respiratory Syndrome (SARS) (2). SARS-CoV-2 primarily spreads through respiratory droplets and close contact, and all populations are susceptible (3). Coronavirus disease 2019 (COVID-19) caused by SARS-CoV-2 was declared by the World Health Organization (WHO) as a public health emergency of international concern on January 30, 2020 and a pandemic on March 12, 2020. As of September 28, 2020, the number of globally confirmed cases was over 32.7 million and the number of mortalities was $>991,000$, according to WHO statistics (4).

As the epidemic progresses, the prevention and control of asymptomatic carriers has gradually become the focus of the global epidemic control effort. For example, at present, China and certain other countries have entered the stage of remission, although sporadic new cases, imported cases and asymptomatic carriers remain risk factors for spreading the virus (5). According to statistics from the National Health Commission of China, there were 375 asymptomatic carriers currently under medical observation by September 29 (6). One study initially estimated that asymptomatic carriers, pre-symptomatic and mild infection of COVID-19 may account for $60 \%$ of all infections (7). In addition, the majority of these patients may not self-isolate, and spread the virus to others unconsciously, as they are unaware that they have been infected with SARS-CoV-2 (8). Therefore, asymptomatic carriers of COVID-19 should be taken seriously because they serve a crucial role in disease transmission. The present review aims to discuss the clinical characteristics and infectivity of asymptomatic carriers and provide suggestions to control these potential hidden sources of infection.

\section{Demographic characteristics}

The studies in the present review identified that the demographic characteristics of asymptomatic carriers are significantly correlated with age. In a study of 147 asymptomatic carriers in Anhui, China, male carriers accounted for $51.7 \%$ and carriers $<20$ years old accounted for $15.6 \%$ (9). Hu et al (10) reported the epidemiological and clinical data of 24 asymptomatic infections with COVID-19, and identified 
that people $<15$ years old were more likely to be asymptomatic carriers compared with patients $>15$ years. The study by Wang et al (11), which reviewed the clinical information of 55 asymptomatic carriers in Shenzhen, China, confirmed that $27 \%$ of asymptomatic carriers were $<18$ years old. Rawat et al (12) confirmed that children were more likely to have asymptomatic infections compared with adults; their data indicated that $27 \%$ of pediatric COVID-19 cases were asymptomatic carriers and that only $7 \%$ of adult patients had no symptoms (12). This result was also verified in Zhejiang, China, where, among 36 children with confirmed COVID-19, $28 \%$ were asymptomatic carriers (13). Tan et al (14) identified that asymptomatic carriers were relatively young (mean age, 34.5 years old). Current evidence suggests that the asymptomatic carriers with SARS-CoV-2 vary by age, however, to the best of our knowledge, no studies have identified differences in sex between asymptomatic carriers.

Previous data may also explain why asymptomatic carriers are more likely to be young people. For example, it may be that the gene expression of angiotensin converting enzyme II (ACE2) in nasal epithelial cells is age-dependent. ACE2 is the host receptor for SARS-CoV-1 and SARS-CoV-2 (15). Bunyavanich et al (16) demonstrated that ACE2 gene expression increased with age, with the lowest expression in younger children $(<10$ years old; $\mathrm{n}=45)$. In addition, Do et al (17) argued that cytokine storms in adults promote stronger inflammatory responses compared with that in younger people. Wu et al (18) also demonstrated that older patients (60-85 years old) and middle-aged patients (40-59 years old) were more likely to induce higher neutralizing antibodies titers compared with younger patients (15-39 years old), which has been shown to be associated with disease severity (19).

\section{Ratio of asymptomatic carriers among all infected persons}

It is not easy to accurately estimate the proportion of asymptomatic carriers among patients with confirmed COVID-19, as the total number of asymptomatic carriers will not be identified unless all people are systematically screened. Moreover, without follow-up observation, it is also difficult to distinguish between pre-symptomatic persons (symptomatic later in the course of the disease) and the asymptomatic carriers (asymptomatic during the whole duration of the disease) (20). Wang et al (21) identified 1,012 patients with non-severe COVID-19 disease with positive reverse transcriptase polymerase chain reaction (RT-PCR) results in Wuhan, China, of which 30 (3.0\%) were asymptomatic (21). In Shanghai, China, another study showed that $3.9 \%$ of the 328 adults diagnosed with COVID-19 were asymptomatic carriers (13 cases) (22). However, a study in Jinan, China, observed that 11 of 47 confirmed patients $(23.4 \%)$ were asymptomatic carriers (23). According to statistics from the Chinese Center for Disease Control and Prevention, on February 11, China recorded a total of 72,314 patients, including 889 asymptomatic cases (1.2\%) (24). However, this statistic was measured prior to the large-scale screening of patients with COVID-19 in China, so the proportion may be less compared with the real data. One study estimated that the rate of asymptomatic carriers was $30.8 \%$ (95\% CI, 7.7-53.8\%) among 565 Japanese individuals evacuated from Wuhan, China (25). There are also studies using published data for statistical model analysis, estimating that the asymptomatic proportion of COVID-19 cases on the Japanese cruise ship Princess Diamond was 17.9\% (95\% CI, 15.5-20.2\%) (26). In a cohort of children, Qiu et al (13) identified that 10 (28\%) of 36 pediatric patients infected with COVID-19 were completely asymptomatic. Similarly, in another study of children with COVID-19, 20 (27\%) of 74 cases were asymptomatic infections (27). In addition, the study by Rawat et al (12), examining patients in the United States of America, demonstrated that $27 \%$ of pediatric patients with COVID-19 had no symptoms, while only $7 \%$ of adult patients had no symptoms (12). Notably, a study conducted in a large hospital in Wuhan, China also found that the asymptomatic infection rate among healthcare workers was $9.7 \%$ (88/908) (28). From these studies, it can be observed that the proportion of asymptomatic carriers varies widely in different studies. In addition, the true prevalence of asymptomatic carriers may be lower compared with current estimates due to the inclusion of pre-symptomatic infections in some cross-sectional studies.

\section{Radiological and laboratory data}

Asymptomatic carriers differ from confirmed patients not only in their symptoms, but also in their laboratory and chest CT results. At present, no uniform pattern in laboratory and radiological manifestations of asymptomatic carriers has been established. Meng et al (29) collected clinical information on 58 asymptomatic patients with COVID-19 pneumonia and identified that all carriers had a normal laboratory and abnormal chest $\mathrm{CT}$ results. The main CT features of asymptomatic patients were ground-glass opacity [(GGO) 55, 94.8\%] at admission. However, among the 24 asymptomatic carriers in Nanjing, China, lymphopenia and leukopenia were not obvious, and chest CT images were normal in the majority of young carriers ( $<15$ years old) (10). Yu and Yang (30) also reported 2 asymptomatic carriers with normal lymphocyte count and chest CT images. Similarly, Wang et al (11) reported increased C-reactive protein and lactate dehydrogenase levels in 10 and 13 carriers, respectively, and only 11 patients (including two with severe infections) exhibited leukopenia, from the follow-up results of 55 asymptomatic carriers (11). A study from Zhou et al (22) followed up 13 cases of asymptomatic carriers, of which 2 patients had leukocytes below the normal range. A recent study identified that 3 of $11(27.3 \%)$ persistent asymptomatic patients with SARS-CoV-2 infection did not have typical chest CT results and the GGO of other carriers had been absorbed in nearly 5 days (31). Ali and Ghonimy (32) examined 44 asymptomatic cases of COVID-19 and identified that the main features of their CT results were GGO (93\%; $n=41)$ and surrounding distribution $(77.3 \%$; $=34)$. In summary, these studies suggest that radiological and laboratory abnormalities vary and laboratory findings and chest CT images can be normal in asymptomatic carriers. Therefore, SARS-CoV-2 infection cannot be ruled out on the basis of normal radiological and laboratory results.

\section{Infectivity and transmission risk of asymptomatic carriers}

A number of studies have confirmed that asymptomatic carriers can transmit the virus. In Anyang, Henan, China, a family cluster of 5 patients with COVID-19 was infected with SARS-CoV-2 
by contact with asymptomatic family members (33). In Nanjing, COVID-19 was also transmitted by asymptomatic carriers to cohabiting family members (10). Yu and Yang (30) described an asymptomatic carrier who had transmitted SARS-CoV-2 to his brother following close contact. Recently, an asymptomatic carrier resulted in 13 secondary cases of infection, reportedly as an asymptomatic super spreader (34). In summary, it is almost unquestionable that asymptomatic carriers have infectivity.

In earlier cross-sectional studies, no significant differences in viral titers and transmission efficiency were identified between asymptomatic and symptomatic patients. A study showed that the viral load in asymptomatic patients was similar to that of symptomatic patients (35). Chen et al (36) followed up and investigated 2,147 close contacts in Ningbo, Zhejiang, China, with a total infection rate of $6.15 \%$, while there was no significant difference in the infection rate between the confirmed cases and asymptomatic cases (6.30 and 4.11\%, respectively) (36). Concomitantly, Yin and Jin (37) analyzed the transmission rate of 157 symptomatic cases and 30 asymptomatic cases of COVID-19 and identified that there was no significant difference in the transmission rate between symptomatic and asymptomatic patients. However, none of these studies excluded pre-symptomatic patients in the incubation period. Another study in Korea including 3 pre-symptomatic patients and 10 asymptomatic carriers suggested that pre-symptomatic patients in the incubation period may have an increased viral load compared with asymptomatic carriers (38). Therefore, the transmission efficiency of asymptomatic carriers estimated by these studies may be increased compared with the real data. Subsequently, increasing evidence suggests that true asymptomatic carriers may have a decreased risk of transmission than symptomatic patients. The study by Liu et al (9) showed that the secondary attack rate of 131 asymptomatic carriers was $2.6 \%$ (24/914), while the secondary attack rate of 16 confirmed cases was $9.7 \%$ (23/236), further demonstrating that the transmission risk of asymptomatic carriers was decreased compared with that of the confirmed cases. In China, a study including 12 asymptomatic carriers in Wuhan also identified that only one carrier has transmitted the virus to a close contact (14). Chen et al (39) tracked 9 asymptomatic carriers and identified that none of them had transmitted SARS-CoV-2 to others, so they speculated that the absence of symptoms such as sneezing and coughing may interfere with virus shedding and decrease the risk of infection. In addition, a systematic review showed that the relative risk of asymptomatic carriers transmission was decreased by $42 \%$ compared with symptomatic transmission, while there were no differences in viral load (40). In light of the data from these studies, asymptomatic carriers may have a weaker transmission efficiency compared with symptomatic patients. However, the transmission ability of asymptomatic carriers cannot be ignored, as some of them may develop into super spreaders. There is no doubt that the early identification of asymptomatic carriers and the prevention of the further spread of the virus are critical to disease control.

\section{Transmission period of asymptomatic carriers}

The study in Korea calculated that the median duration of virus shedding (from the first confirmed positive RNA test result to the first negative RNA test result) in asymptomatic carriers was 4.5 days (2.5-9 days), and all asymptomatic carriers were almost non-infectious after isolation of 14 days (38). However, the study from $\mathrm{Hu}$ et al (10) showed that the median spread interval was 9.5 days (up to 21 days), and the actual infection period may be longer as the exact date of the first infection is uncertain for some carriers. Tan et al (14) demonstrated that the median duration of virus shedding was 11.5 (9-14) days among 12 carriers in Wuhan, 2 of which were found to have positive RNA results lasting up to 2 months (14). Long et al (41) also reported a median duration of viral shedding of 19 (15-26) days in 37 asymptomatic carriers. Meanwhile, Pan et al (42) retrospectively analyzed 26 persistently asymptomatic SARS-CoV-2 carriers and identified that the median time from diagnosis to negative nucleic acid testing was 7.5 days (2-20 days) for carriers with normal or atypical chest CT findings, compared with 12.5 days (8-22 days) for those with typical GGO (42). These studies have shown that SARS-CoV-2 can remain present in asymptomatic carriers for a long period of time as long as 2 months and carriers with abnormal chest CT findings may have a longer transmission period.

\section{Advice to identify asymptomatic carriers}

Asymptomatic carriers can transmit the virus as an unseen source of infection, which is a great challenge for epidemic prevention and control, so the importance of implementing social distancing measures and using masks cannot be overemphasized. It is also of vital importance to identify and isolate asymptomatic carriers at an early stage. The question of how asymptomatic carriers can be identified earlier remains. At present, the detection of SARS-CoV-2 virus infection mainly depends on nucleic acid, serum antibody and chest CT. As the chest CT images of some asymptomatic carriers are entirely normal, particularly in young people, the present study only discussed the detection of nucleic acids and antibodies. Studies have shown that nucleic acid detection has the disadvantages of high false-negative rate and low sensitivity, although it is widely used $(28,43,44)$. The positive rates of RT-PCR detection of sputum specimens, nasal swabs and throat swabs were 74.4-88.9, 53.6-73.3 and 29.6-61.3\%, respectively (44). In contrast, the sensitivity and specificity of the rapid $\operatorname{IgM}-\operatorname{IgG}$ combined antibody test were 88.7 and $90.6 \%$, respectively (43). There is also evidence that the sensitivity of total antibodies is increased compared with that of $\operatorname{IgM}$ or $\operatorname{IgG}$ alone (45). A study identified that the asymptomatic infection rate confirmed by RT-PCR alone was $4.2 \%$ (38/908), while combined with RT-PCR test and serological detection was 9.7\% (88/908) (28).

Considering the available data, the combination of serum antibody testing and nucleic acid testing is recommended to identify asymptomatic carriers in high-risk areas or high-risk populations. Confirmed asymptomatic carriers should be subject to centralized quarantine and observation for at least 14 days until they receive 2 consecutive negative nucleic acid test results (at least $24 \mathrm{~h}$ apart).

\section{Conclusions}

Based on the literature, the present review identified that the proportion of asymptomatic carriers of SARS-CoV-2 in all 
infections varies significantly between different studies, but asymptomatic infection may be correlated with age. Among the young population, the proportion of asymptomatic carriers may be increased. There is no uniform pattern of laboratory and radiological findings in asymptomatic carriers, and some asymptomatic carriers may have completely normal chest CT and laboratory results. It is almost certain that asymptomatic carriers can transmit SARS-CoV-2, but they may have a decreased risk of transmission compared with symptomatic patients. At present, the management of asymptomatic carriers should become a focus of epidemic prevention, particularly in countries with stable epidemics. Dual detection of serum antibodies and nucleic acids in high-risk areas and high-risk groups and quarantining for at least 14 days is recommended following diagnosis as an asymptomatic carrier.

\section{Acknowledgements}

Not applicable.

\section{Funding}

No funding was received.

\section{Availability of data and materials}

Not applicable.

\section{Authors' contributions}

WZ and FX made significant contributions to select the topic of the review. WZ was responsible for the collection and collation of documents and writing draft manuscripts. HZ, FX and XW revised the writing of the manuscript. All authors read and approved the final manuscript.

\section{Ethics approval and consent to participate}

Not applicable.

\section{Patient consent for publication}

Not applicable.

\section{Competing interests}

The authors declare that they have no competing interests.

\section{References}

1. Huang C, Wang Y, Li X, Ren L, Zhao J, Hu Y, Zhang L, Fan G $\mathrm{Xu} \mathrm{J}, \mathrm{Gu} \mathrm{X}$, et al: Clinical features of patients infected with 2019 novel coronavirus in Wuhan, China. Lancet 395: 497-506, 2020.

2. Guan WJ, Ni ZY, Hu Y, Liang WH, Ou CQ, He JX, Liu L, Shan H, Lei CL, Hui DSC, et al: Clinical Characteristics of Coronavirus Disease 2019 in China. N Engl J Med 382: 1,708-1,720, 2020.

3. Jin YH, Cai L, Cheng ZS, Cheng H, Deng T, Fan YP, Fang C, Huang D, Huang LQ, Huang Q, et al: A rapid advice guideline for the diagnosis and treatment of 2019 novel coronavirus (2019-nCoV) infected pneumonia (standard version). Mil Med Res 7: 4, 2020.
4. WHO: Coronavirus disease 2019 (COVID-19) Situation Report. https://www.who.int/emergencies/diseases/novel-coronavirus2019/situation-reports/. Updated September 28, 2020

5. Zhang J, Wu S and Xu L: Asymptomatic carriers of COVID-19 as a concern for disease prevention and control: More testing, more follow-up. Biosci Trends 14: 206-208, 2020.

6. China NHCtPsRo: The latest updates on COVID-19 outbreaks as of 24:00 on September 29. http://www.nhc.gov.cn/xcs/yqtb/ 202009/9015bdbd354147919d82bd84a68cd919.shtml. Updated September 29, 2020

7. Qiu J: Covert coronavirus infections could be seeding new outbreaks. Nature:Mar. 20,2020 (Epub ahead of print). doi: 10.1038/ d41586-020-00822-x.

8. Al-Sadeq DW and Nasrallah GK: The incidence of the novel coronavirus SARS-CoV-2 among asymptomatic patients: A systematic review. Int J Infect Dis 98: 372-380, 2020.

9. Liu Z, Chu R, Gong L, Su B and Wu J: The assessment of transmission efficiency and latent infection period in asymptomatic carriers of SARS-CoV-2 infection. Int J Infect Dis 99: 325-327, 2020.

10. Hu Z, Song C, Xu C, Jin G, Chen Y, Xu X, Ma H, Chen W, Lin Y, Zheng Y, et al: Clinical characteristics of 24 asymptomatic infections with COVID-19 screened among close contacts in Nanjing, China. Sci China Life Sci 63: 706-711, 2020.

11. Wang Y, Liu Y, Liu L, Wang X, Luo N and Li L: Clinical Outcomes in 55 patients with severe acute respiratory syndrome coronavirus 2 Who Were asymptomatic at hospital admission in shenzhen, China. J Infect Dis 221: 1,770-1,774, 2020.

12. Rawat M, Chandrasekharan P,Hicar MD and Lakshminrusimha S: COVID-19 in newborns and infants-low risk of severe disease: Silver Lining or Dark Cloud? Am J Perinatol 37: 845-849, 2020.

13. Qiu H, Wu J, Hong L, Luo Y, Song Q and Chen D: Clinical and epidemiological features of 36 children with coronavirus disease 2019 (COVID-19) in Zhejiang, China: An observational cohort study. Lancet Infect Dis 20: 689-696, 2020.

14. Tan F, Wang K, Liu J, Liu D, Luo J and Zhou R: Viral Transmission and Clinical Features in Asymptomatic Carriers of SARS-CoV-2 in Wuhan, China. Front Med (Lausanne) 7: 547, 2020.

15. Tai W, He L, Zhang X, Pu J, Voronin D, Jiang S, Zhou Y and Du L: Characterization of the receptor-binding domain (RBD) of 2019 novel coronavirus: Implication for development of RBD protein as a viral attachment inhibitor and vaccine. Cell Mol Immunol 17: 613-620, 2020.

16. Bunyavanich S, Do A and Vicencio A: Nasal gene expression of angiotensin-converting enzyme 2 in children and adults. JAMA 23: 2427-2429, 2020.

17. Do LAH, Anderson J, Mulholland EK and Licciardi PV: Can data from paediatric cohorts solve the COVID-19 puzzle? PLoS Pathog 16: e1008798, 2020.

18. Wu F, Wang A, Liu M, Wang Q, Chen J, Xia S, Ling Y, Zhang Y, Xun J, Lu L, et al: Neutralizing antibody responses to SARS-CoV-2 in a COVID-19 recovered patient cohort and their implications. medRxiv: Apr. 20, 2020 (Epub ahead of print). doi: https://doi.org/10.1101/2020.03.30.20047365.

19. Casadevall A and Pirofski LA: The convalescent sera option for containing COVID-19. J Clin Invest 130: 1545-1548, 2020.

20. Arteaga-Livias K, Pecho-Silva S, Panduro-Correa V and Rodríguez-Morales AJ: The dilemmas of the classification of SARS-CoV-2 infection without clinical manifestations: Asymptomatic or presymptomatic. J Formos Med Assoc 119: $1237-1238,2020$

21. Wang X, Fang J, Zhu Y, Chen L, Ding F, Zhou R, Ge L, Wang F, Chen Q, Zhang Y and Zhao Q: Clinical characteristics of non-critically ill patients with novel coronavirus infection (COVID-19) in a Fangcang Hospital. Clin Microbiol Infect 26: $1,063-1,068,2020$

22. Zhou X, Li Y, Li T and Zhang W: Follow-up of asymptomatic patients with SARS-CoV-2 infection. Clin Microbiol Infect 26: 957-959, 2020

23. Ma Y, Xu QN, Wang FL, Ma XM, Wang XY, Zhang XG and Zhang ZF: Characteristics of asymptomatic patients with SARS-CoV-2 infection in Jinan, China. Microbes Infect 22: 212-217, 2020.

24. Epidemiology Working Group for NCIP Epidemic Response, Chinese Center for Disease Control and Prevention. The epidemiological characteristics of an outbreak of 2019 novel coronavirus diseases (COVID-19) in China. Zhonghua Liu Xing Bing Xue Za Zhi 41: 145-151, 2020 (In Chinese). 
25. Nishiura H, Kobayashi T, Miyama T, Suzuki A, Jung SM, Hayashi K, Kinoshita R, Yang Y, Yuan B, Akhmetzhanov AR and Linton NM: Estimation of the asymptomatic qratio of novel coronavirus infections (COVID-19). Int J Infect Dis 94: 154-155, 2020.

26. Mizumoto K, Kagaya K, Zarebski A and Chowell G: Estimating the asymptomatic proportion of coronavirus disease 2019 (COVID-19) cases on board the Diamond Princess cruise ship, Yokohama, Japan, 2020. Euro Surveill 25: 2000180, 2020.

27. Wu Q, Xing Y, Shi L, Li W, Gao Y, Pan S, Wang Y, Wang W and Xing Q: Coinfection and other clinical characteristics of COVID-19 in children. Pediatrics 146: e20200961, 2020.

28. Zhao D, Wang M, Wang M, Zhao Y, Zheng Z, Li X, Zhang Y, Wang T, Zeng S, Hu W, et al: Asymptomatic infection by SARS-CoV-2 in healthcare workers: A study in a large teaching hospital in Wuhan, China. Int J Infect Dis 99: 219-225, 2020.

29. Meng H, Xiong R, He R, Lin W, Hao B, Zhang L, Lu Z, Shen X, Fan T, Jiang W, et al: CT imaging and clinical course of asymptomatic cases with COVID-19 pneumonia at admission in Wuhan, China. J Infect 81: e33-e39, 2020.

30. Yu X and Yang R: COVID-19 transmission through asymptomatic carriers is a challenge to containment. Influenza Other Respir Viruses 14: 474-475, 2020.

31. Yan S, Chen H, Xie RM, Guan CS, Xue M, Lv ZB, Wei LG, Bai Y and Chen BD: Chest CT evaluation of 11 persistent asymptomatic patients with SARS-CoV-2 infection. Jpn J Infect Dis: Jun 30, 2020 (Epub ahead of print). doi: 10.7883/yoken.JJID.2020.264.

32. Ali RMM and Ghonimy MBI: Radiological findings spectrum of asymptomatic coronavirus (COVID-19) patients. Egypt J Radiol Nucl Med 51: 156, 2020.

33. Bai Y, Yao L, Wei T, Tian F, Jin DY, Chen L and Wang M: Presumed Asymptomatic Carrier Transmission of COVID-19. JAMA 323: 1406-1407, 2020.

34. Yu X, Ran D, Wang J, Qin Y, Liu R, Shi X, Wang Y, Xie C, Jiang $\mathrm{J}$ and Zhou J: Unclear but present danger: An asymptomatic SARS-CoV-2 carrier. Genes Dis: Jul 23, 2020 (Epub ahead of print). doi: 10.1016/j.gendis.2020.07.010.

35. Zou L, Ruan F, Huang M, Liang L, Huang H, Hong Z, Yu J, Kang M, Song Y, Xia J, et al: SARS-CoV-2 viral load in upper respiratory specimens of infected patients. N Engl J Med 382: $1177-1179,2020$

36. Chen Y, Wang AH, Yi B, Ding KQ, Wang HB, Wang JM, Shi HB, Wang SJ and Xu GZ: Epidemiological characteristics of infection in COVID-19 close contacts in Ningbo city. Zhonghua Liu Xing Bing Xue Za Zhi 41: 667-671, 2020 (In Chinese).
37. Yin $\mathrm{G}$ and Jin $\mathrm{H}$ : Comparison of transmissibility of coronavirus between symptomatic and asymptomatic patients: Reanalysis of the ningbo COVID-19 Data. JMIR Public Health Surveill 6: e19464, 2020

38. Kim SE, Jeong HS, Yu Y, Shin SU, Kim S, Oh TH, Kim UJ, Kang SJ, Jang HC, Jung SI and Park KH: Viral kinetics of SARS-CoV-2 in asymptomatic carriers and presymptomatic patients. Int J Infect Dis 95: 441-443, 2020.

39. Chen F, Fu D, Yang Q, Geng Z, Xia J, Wang Z and Wang L: Low transmission risk of 9 asymptomatic carriers tested positive for both SARS-CoV-2 nucleic acid and serum IgG. J Infect 81: 452-482, 2020

40. Byambasuren O, Cardona M, Bell K, Clark J, McLaws ML and Glasziou P: Estimating the extent of asymptomatic COVID-19 and its potential for community transmission: Systematic review and meta-analysis. medRxiv: Sep 23, 2020 (Epub ahead of print) doi: https://doi.org/10.1101/2020.05.10.20097543.

41. Long QX, Tang XJ, Shi QL, Li Q, Deng HJ, Yuan J, Hu JL, Xu W, Zhang Y, Lv FJ, et al: Clinical and immunological assessment of asymptomatic SARS-CoV-2 infections. Nat Med 26: 1200-1204, 2020.

42. Pan Y, Yu X, Du X, Li Q, Li X, Qin T, Wang M, Jiang M, Li J, Li W, et al: Epidemiological and clinical characteristics of 26 asymptomatic severe acute respiratory syndrome coronavirus 2 carriers. J Infect Dis 221: 1,940-1,947, 2020.

43. Lu S, Lin J, Zhang Z, Xiao L, Jiang Z, Chen J, Hu C and Luo S: Alert for non-respiratory symptoms of coronavirus disease 2019 patients in epidemic period: A case report of familial cluster with three asymptomatic COVID-19 patients. J Med Virol: Mar 19, 2020 (Epub ahead of print). doi: https://doi.org/10.1002/jmv.25776.

44. Yang Y, Yang M, Shen C, Wang F, Yuan J, Li J, Zhang M, Wang Z, Xing L, Wei J, et al: Evaluating the accuracy of different respiratory specimens in the laboratory diagnosis and monitoring the viral shedding of 2019-nCoV infections. medRxiv: doi: https:// doi.org/10.1101/2020.02.11.20021493.

45. Zhao J, Yuan Q, Wang H, Liu W, Liao X, Su Y, Wang X, Yuan J, $\mathrm{Li} \mathrm{T,} \mathrm{Li} \mathrm{J,} \mathrm{et} \mathrm{al:} \mathrm{Antibody} \mathrm{responses} \mathrm{to} \mathrm{SARS-CoV-2} \mathrm{in} \mathrm{patients}$ of novel coronavirus disease 2019. Clin Infect Dis 71: 2027-2034, 2020 .

This work is licensed under a Creative Commons Attribution-NonCommercial-NoDerivatives 4.0 International (CC BY-NC-ND 4.0) License. 